Sādhanā Vol. 40, Part 2, April 2015, pp. 369-377. (C) Indian Academy of Sciences

\title{
On the solution of a class of fuzzy system of linear equations
}

\section{DAVOD KHOJASTEH SALKUYEH}

Faculty of Mathematical Sciences, University of Guilan, P.O. Box 1914, Rasht, Iran

e-mail: khojasteh@guilan.ac.ir; salkuyeh@gmail.com

MS received 13 May 2014; revised 18 July 2014; accepted 1 November 2014

Abstract. Inthis paper, we consider the system of linear equations $A \tilde{x}=\tilde{b}$, where $A \in \mathbb{R}^{n \times n}$ is a crisp H-matrix and $\tilde{b}$ is a fuzzy $n$-vector. We then investigate the existence and uniqueness of a fuzzy solution to this system. The results can also be used for the class of M-matrices and strictly diagonally dominant matrices. Finally, some numerical examples are given to illustrate the presented theoretical results.

Keywords. Fuzzy; system of linear equations; M-matrix; H-matrix; strictly diagonally dominant.

\section{Introduction}

Systems of linear equations play a major role in various field of science and engineering. In many applications, some of the system parameters are represented by fuzzy numbers rather than crisp numbers. Therefore, developing mathematical models and numerical procedures for the fuzzy system of linear equations would be of interest. Friedman et al (1998) considered a general model for solving the fuzzy system of linear equations (hereafter denoted by FSLE)

$$
A \tilde{x}=\tilde{b}
$$

where the coefficient matrix is crisp and its right-hand side is an arbitrary fuzzy vector. They stated some conditions for the existence of a unique fuzzy solution to FSLE by using the embedding method (Friedman et al 1998) and converting the original system to a crisp linear system of equations. Later, several authors studied FSLE. Allahviranloo (2004), used the Jacobi and Gauss-Seidel iterative methods to compute an approximate solution to Eq. (1). He also used the successive overrelaxation iterative method for solving FSLE in (Allahviranloo 2005). Dehghan \& Hashemi (2006) investigated the existence of a solution to Eq. (1) provided that the matrix $A$ is strictly diagonally dominant matrix with positive diagonal entries and then applied several 
iterative methods for solving FSLE. Hashemi et al (2008) have studied FSLE under the condition that the coefficient matrix is a crisp M-matrix. Some block iterative methods to solve FSLE have been presented by Wang \& Zheng (2007).

Generalization of FSLE solvers to fully FSLE (FFSLE), where both of the system coefficient matrix and the right-hand side vector are fuzzy, is not a straightforward task. Dehghan et al (2006) developed some direct methods such as Cramer's rule, Gaussian elimination method, LU factorization and linear programming approach to solve FFSLE. Then, they applied some iterative methods to solve FFSLE (Dehghan et al 2007). Several papers have been presented in the literature to develop the existing methods for solving FFLSE or fully fuzzy matrix equations (e.g., see Allahviranloo et al 2008; Mosleh et al 2009; Nasseri et al 2008; Nasseri \& Sohrabi 2010, Nasseri \& Zahmatkesh 2010; Senthilkumar \& Rajendran 2011 and references therein). Recently, Babbar et al (2013a) have presented three new effective computational methods to solve FFSLE involving triangular fuzzy numbers. The proposed methods are based on analytical decomposition of a fuzzy linear system into its equivalent crisp form. They also proposed a new method based on linear programming to solve a general fully fuzzy matrix equation in (Babbar et al 2013b). More recently, Gong et al (2014) proposed a simple and practical approach for solving the dual fuzzy matrix equation.

In this paper, we consider the FSLE (1) where $A \in \mathbb{R}^{n \times n}$ is a crisp H-matrix. We then investigate the existence and uniqueness of a solution to FSLE. To do so, we first review some notations, definitions and results that will be used in the next sections.

The vector $x=\left(x_{1}, x_{2}, \ldots, x_{n}\right)^{T} \in \mathbb{R}^{n}$ is said to be positive (non-negative), if $x_{i}>0$ $\left(x_{i} \geq 0\right), i=1,2, \ldots, n$. In this case, we write $x>0(x \geq 0)$. Similar definitions can be written for matrices. A permutation matrix is a matrix that has exactly one 1 in each row or column and 0 s elsewhere. It is easy to show that if $P$ is a permutation matrix, then $P$ is non-singular and $P^{-1}=P^{T}$.

Definition 1 (Axelsson 1996) A matrix $A=\left(a_{i j}\right) \in \mathbb{R}^{n \times n}$ is said to be an M-matrix if $a_{i i}>0$ for $i=1, \ldots, n, a_{i j} \leq 0$, for $i \neq j, A$ is non-singular and $A^{-1} \geq 0$. The comparison matrix of $A$, denoted by $\mathcal{M}(A)=\left(m_{i j}\right) \in \mathbb{R}^{n \times n}$, is defined by

$$
m_{i j}=\left\{\begin{aligned}
\left|a_{i i}\right|, & \text { if } i=j \\
-\left|a_{i j}\right|, & \text { if } i \neq j .
\end{aligned}\right.
$$

$A$ is called an H-matrix if $\mathcal{M}(A)$ is an M-matrix.

Definition 2 (Axelsson 1996) A matrix $A=\left(a_{i j}\right) \in \mathbb{R}^{n \times n}$ is said to be generalized strictly diagonally dominant if

$$
\left|a_{i i}\right| x_{i}>\sum_{\substack{j=1 \\ j \neq i}}^{n}\left|a_{i j}\right| x_{j}, \quad i=1,2, \ldots, n
$$

for some positive vector $x=\left(x_{1}, x_{2}, \ldots, x_{n}\right)^{T} . A$ is called strictly diagonally dominant if Eq. (2) is valid for $x=(1,1, \ldots, 1)^{T}$. 
Theorem 1 (Axelsson 1996, Lemma 6.4) A matrix $A \in \mathbb{R}^{n \times n}$ is an H-matrix if and only if $A$ is generalized strictly diagonally dominant.

Clearly, every H-matrix is non-singular and every M-matrix is an H-matrix. From Theorem 2, it is easy to see that each strictly diagonally dominant matrix is an H-matrix.

This paper is organized as follows. In section 2, some basic definitions and results on FSLE are given. Our main results are drawn in section 3. Section 4 is devoted to some numerical examples illustrating the theoretical results. Some concluding remarks are given in section 5 .

\section{Preliminaries}

In this section, we review some of the basic notations of fuzzy number arithmetic and fuzzy system of linear equations.

Definition 3 (Ma et al 2000): A fuzzy number is a fuzzy set $\tilde{u}: \mathbb{R} \rightarrow I=[0,1]$ which satisfies

(a) $\tilde{u}$ is upper semi-continuous,

(b) $\tilde{u}(x)=0$ outside some interval $[c, d]$,

(c) There are real numbers $a$ and $b$ such that $c \leq a \leq b \leq d$ and

- $\tilde{u}$ is monotonic increasing on $[c, a]$,

- $\tilde{u}$ is monotonic decreasing on $[b, d]$,

- $\tilde{u}(x)=1$ for $x \in[a, b]$.

Definition 4 (Friedman et al 1998): A fuzzy number $\tilde{u}$ in parametric form is defined an ordered pair of functions $(\underline{u}(r), \bar{u}(r)), 0 \leq r \leq 1$, which satisfies the following requirements

(a) $\underline{u}(r)$ is a bounded left continuous non-decreasing function over $[0,1]$,

(b) $\overline{\bar{u}}(r)$ is a bounded right continuous non-increasing function over [0,1],

(c) $\underline{u}(r) \leq \bar{u}(r), 0 \leq r \leq 1$.

A crisp number $\alpha$ is represented by $\underline{u}(r)=\bar{u}(r)=\alpha, 0 \leq r \leq 1$. A popular representation for fuzzy number is the trapezoidal representation $\tilde{u}=\left(x_{0}, y_{0}, \alpha, \beta\right)$ with defuzzifier interval $\left[x_{0}, y_{0}\right]$, left fuzziness $\alpha$ and right fuzziness $\beta$ (Hashemi et al 2008). The membership function of this trapezoidal number is as follows:

$$
\tilde{u}(x)= \begin{cases}\frac{1}{\alpha}\left(x-x_{0}+\alpha\right), & x_{0}-\alpha \leq x \leq x_{0}, \\ 1, & x_{0} \leq x \leq y_{0} \\ \frac{1}{\beta}\left(y_{0}-x+\beta\right), & y_{0} \leq x \leq y_{0}+\beta \\ 0, & \text { otherwise }\end{cases}
$$

The parametric form of this number is

$$
\underline{u}(r)=x_{0}-\alpha+\alpha r, \quad \bar{u}(r)=y_{0}+\beta-\beta r .
$$

When $x_{0}=y_{0}$, a trapezoidal fuzzy number is reduced to a triangular fuzzy number. 
To introduce the FSLE and defining its solution, we recall the arithmetic operations of arbitrary fuzzy numbers $\tilde{x}=(\underline{x}(r), \bar{x}(r))$ and $\tilde{y}=(y(r), \bar{y}(r)), 0 \leq r \leq 1$ and real number $k$ as follows

(a) $\tilde{x}=\tilde{y}$ if and only if $\underline{x}(r)=\underline{y}(r)$ and $\bar{x}(r)=\bar{y}(r)$;

(b) $\tilde{x}+\tilde{y}=(\underline{x}(r)+\underline{y}(r), \bar{x}(r)+\bar{y}(r))$;

(c) $k \tilde{x}= \begin{cases}(k \underline{x}(r), k \bar{x}(r)), & k \geq 0, \\ (k \bar{x}(r), k \underline{x}(r)), & k<0 .\end{cases}$

Definition 5 (Friedman et al 1998): A fuzzy number vector $\tilde{x}=\left(\tilde{x}_{1}, \tilde{x}_{2}, \ldots, \tilde{x}_{n}\right)^{T}$, where $\tilde{x}_{i}=$ $\left(x_{i}(r), \overline{x_{i}}(r)\right), 0 \leq r \leq 1, i=1,2, \ldots, n$, is called a solution to FSLE (1) if

$$
\left\{\begin{array}{l}
\underline{\sum_{j=1}^{n} a_{i j} x_{j}}=\sum_{j=1}^{n} \underline{a_{i j} x_{j}}=\underline{y_{i}}, \\
\overline{\sum_{j=1}^{n} a_{i j} x_{j}}=\sum_{j=1}^{n} \overline{a_{i j} x_{j}}=\overline{y_{i}},
\end{array} \quad i=1,2, \ldots, n .\right.
$$

It is easy to see that Eq. (3) is equivalent to the $m \times m(m=2 n)$ crisp system of linear equations

$$
\left(\begin{array}{ll}
S_{1} & S_{2} \\
S_{2} & S_{1}
\end{array}\right)\left(\frac{X}{\bar{X}}\right)=\left(\frac{Y}{\bar{Y}}\right),
$$

where $S_{1}=\left(\alpha_{i j}\right) \in \mathbb{R}^{m \times m}$ in which

$$
\alpha_{i j}=\left\{\begin{array}{cc}
a_{i j}, & \text { if } a_{i j}>0, \\
0, & \text { otherwise, }
\end{array}\right.
$$

and $S_{2}=A-S_{1}$. Moreover, in Eq. (4) we have $\underline{Y}=\left(\underline{y}_{1}, \underline{y}_{2}, \ldots, \underline{y}_{n}\right)^{T}, \bar{Y}=\left(\bar{y}_{1}, \bar{y}_{2}, \ldots, \bar{y}_{n}\right)^{T}$, $\underline{X}=\left(\underline{x}_{1}, \underline{x}_{2}, \ldots, \underline{x}_{n}\right)^{T}$ and $\bar{X}=\left(\bar{x}_{1}, \bar{x}_{2}, \ldots, \bar{x}_{n}\right)^{T}$.

Theorem 2 (Friedman et al (1998), Theorem 1): The coefficient matrix in Eq. (4) is non-singular if and only if $A=S_{1}+S_{2}$ and $S_{1}-S_{2}$ are both non-singular.

Theorem 3 (Friedman et al (1998), Theorem 3): The components of the unique solution X of Eq. (4) represent a solution fuzzy vector to the system (1) for arbitrary $Y$ if and only if $S^{-1}$ is nonnegative, i.e., $S^{-1} \geq 0$.

\section{Main results}

In this section, we investigate the existence and uniqueness of a fuzzy solution to (1) when its coefficient matrix is an H-marix. For convenience we denote Eq. (4) by $S X=Y$. We now state and prove the main theorem of the paper. 
Theorem 4: If the coefficient matrix of system (1) is an H-matrix, then there exists a permutation matrix $P$ such that $P S$ is also an H-matrix.

Proof: Let $A$ be an H-matrix. We construct a permutation matrix $P=\left(p_{i j}\right)$ such that $\hat{S}=P S$ is an H-matrix. To do so, we first set $P=I$, where $I$ is the identity matrix of dimension $m$, and then modify some of its entries as follows

$$
\left\{\begin{array}{l}
\text { For } i=1, \ldots, n, \text { if } S_{i i}=0, \text { then } P_{i i}:=0 \text { and } P_{i, i+n}:=1 \\
\text { For } i=n+1, \ldots, m, \text { if } S_{i i}=0, \text { then } P_{i i}:=0 \text { and } P_{i, i-n}:=1
\end{array}\right.
$$

We claim that $P$ is the desired permutation matrix. Since $A$ is an H-matrix, according to Theorem 1 there exists a positive vector $x=\left(x_{1}, x_{2}, \ldots, x_{n}\right)^{T} \in \mathbb{R}^{n}$ such that

$$
\left|a_{i i}\right| x_{i}>\sum_{\substack{j=1 \\ j \neq i}}^{n}\left|a_{i j}\right| x_{j}, \quad i=1,2, \ldots, n
$$

Let

$$
\hat{x}=\left(x^{T}, x^{T}\right)^{T}=\left(\hat{x}_{1}, \ldots, \hat{x}_{n}, \hat{x}_{n+1}, \ldots, \hat{x}_{m}\right)^{T} .
$$

Obviously, we have $\hat{x}>0$. It is not difficult to show that

$$
\begin{cases}\left|\hat{s}_{i i}\right| \hat{x}_{i}=\left|a_{i i}\right| x_{i}>\sum_{\substack{j=1 \\ j \neq i}}^{n}\left|a_{i j}\right| x_{j}=\sum_{\substack{j=1 \\ j \neq i}}^{m}\left|\hat{s}_{i j}\right| \hat{x}_{j}, & \text { for } i=1, \ldots, n ; \\ \left|\hat{s}_{i i}\right| \hat{x}_{i}=\left|a_{i-n, i-n}\right| x_{i-n}>\sum_{\substack{j=1 \\ j \neq i-n}}^{n}\left|a_{i-n, j}\right| x_{j}=\sum_{\substack{j=1 \\ j \neq i}}^{m}\left|\hat{s}_{i j}\right| \hat{x}_{j}, & \text { for } i=n+1, \ldots, m,\end{cases}
$$

where $\hat{s}_{i j}$ 's are the entries of $\hat{S}$. This shows that $\hat{S}$ is an H-matrix.

Corollary 1 (a): If the coefficient matrix of system (1) is an H-matrix with positive diagonal entries, then $S$ is also an H-matrix.

Corollary 1 (b): If the coefficient matrix of system (1) is an M-matrix, then $S$ is also an M-matrix.

Proof (a): By refereing to Eq. (5), we see that if $A$ is an H-matrix with positive diagonal entries then $P=I$, where $I$ is the identity matrix. Therefore, $P S=S$ and as a result $P S$ is an H-matrix.

Proof (b): Diagonal entries of an M-matrix are positive. On the other hand, every M-matrix is an H-matrix. Therefore, according to part (a), $P=I$ and as a result $P S=S$. Obviously, diagonal entries of $S$ are positive and its offdiagonal entries are nonpositive. Therefore, we conclude that the matrix $S$ is an M-matrix. 
Corollary 2 (a): If the coefficient matrix of system (1) is strictly diagonally dominant, then there is a permutation matrix $P$ such that $P S$ is also strictly diagonally dominant.

Corollary 2 (b): If the coefficient matrix of system (1) is strictly diagonally dominant with positive diagonal entries, then $S$ is also strictly diagonally dominant.

Proof: This corollary is an immediate result of the second part of Definition 2 together with Theorem 4 and Corollary 1.

Some important observations can be posed here. From the presented theoretical results, we see that if the matrix $A$ has any properties of being H-matrix, M-matrix or strictly diagonally dominant then there is a permutation matrix $P$ (in the case that the diagonal entries of $A$ are positive then we have $P=I$ ) such that $P S$ has the same property as $A$ and in any of these cases the matrix $P S$ is non-singular. Since $P$ is non-singular, we can deduce that the matrix $S$ is non-singular and the system $S X=Y$ has the unique solution $X^{*}=S^{-1} Y$.

If $A$ is an H-matrix or strictly diagonally dominant matrix then it does not guarantee for the computed solution to Eq. (1) being a fuzzy vector. However, when the coefficient matrix of Eq. (1) is a M-matrix, according to the second part of Corollary 1, $S$ is an M-matrix and we have $S^{-1} \geq 0$. Therefore, by Theorem 3the solution of system (1) is a fuzzy vector. Moreover, as mentioned in (Salkuyeh 2011), when $A$ is an M-matrix then $S$, can be partitioned as

$$
S=\left(\begin{array}{cc}
D & E \\
E & D
\end{array}\right)
$$

where $D=\operatorname{diag}\left(a_{11}, a_{22}, \ldots, a_{n n}\right)$ and $E=A-D$. Therefore several iterative methods such as the AOR iterative method (Hadjidimos 1978; Salkuyeh 2011), or direct methods such the LU factorization of $S$ can be exploited to solve the system $S X=Y$. The book (Meurant 1999) is a comprehensive reference for different methods to solve such systems.

The last comment which we present here is due to the study of a method to solve the more general case that the coefficient matrix $A$ is an H-matrix. If $A$ is an H-matrix, then by Theorem 4 the matrix $\hat{S}$ is an H-matrix. It is well-known that if $A$ is an H-matrix, then its LU factorization exists (Meurant 1999, Theorem 2.17). Therefore, one can solve $\hat{S} X=P Y$ instead of $S X=Y$. In fact, since $\hat{S}$ is an H-matrix, we can use its LU factorization to solve $\hat{S} X=P Y$. In this paper, we use this method for the presented examples.

\section{Numerical examples}

In this section, some numerical experiments are given to illustrate the theoretical results presented in this paper. The triangular fuzzy numbers are used in all of the following numerical examples.

Example 1: In the first example, we consider the FSLE $A \tilde{x}=\tilde{b}$, where

$$
A=\left(\begin{array}{ccc}
2 & -3 & 1 \\
-1 & -3 & -1 \\
-1 & 2 & 5
\end{array}\right), \quad \tilde{b}=\left(\begin{array}{c}
(-11+9 r, 7-9 r) \\
(-22+8 r,-6-8 r) \\
(9+10 r, 29-10 r)
\end{array}\right)
$$


It is easy to see that $A$ is an H-matrix. The corresponding matrix $S$ and its associated permutation matrix $P$ are

$$
S=\left(\begin{array}{cccccc}
2 & 0 & 1 & 0 & -3 & 0 \\
0 & 0 & 0 & -1 & -3 & -1 \\
0 & 2 & 5 & -1 & 0 & 0 \\
0 & -3 & 0 & 2 & 0 & 1 \\
-1 & -3 & -1 & 0 & 0 & 0 \\
-1 & 0 & 0 & 0 & 2 & 5
\end{array}\right), \quad P=\left(\begin{array}{llllll}
1 & 0 & 0 & 0 & 0 & 0 \\
0 & 0 & 0 & 0 & 1 & 0 \\
0 & 0 & 1 & 0 & 0 & 0 \\
0 & 0 & 0 & 1 & 0 & 0 \\
0 & 1 & 0 & 0 & 0 & 0 \\
0 & 0 & 0 & 0 & 0 & 1
\end{array}\right)
$$

We have

$$
\hat{S}=P S=\left(\begin{array}{cccccc}
2 & 0 & 1 & 0 & -3 & 0 \\
-1 & -3 & -1 & 0 & 0 & 0 \\
0 & 2 & 5 & -1 & 0 & 0 \\
0 & -3 & 0 & 2 & 0 & 1 \\
0 & 0 & 0 & -1 & -3 & -1 \\
-1 & 0 & 0 & 0 & 2 & 5
\end{array}\right)
$$

which is an H-matrix. We see that Theorem 4 holds good here. Therefore, we solve the system $\hat{S} X=P Y$, by using the LU factorization of $\hat{S}$. This results in the exact solution of the system as

$$
\tilde{x}^{*}=\left(\begin{array}{l}
(1+r, 3-r) \\
(1+2 r, 5-2 r) \\
(2+r, 4-r)
\end{array}\right) \text {. }
$$

Example 2: In this example, we consider the FSLE $A \tilde{x}=\tilde{b}$, where

$$
A=\left(\begin{array}{lll}
2 & -1 & 1 \\
-3 & 5 & 1 \\
-2 & -3 & 4
\end{array}\right), \quad \tilde{b}=\left(\begin{array}{l}
(-2+9 r, 13-6 r) \\
(-7+12 r, 25-20 r) \\
(-23+14 r, 4-13 r)
\end{array}\right)
$$

Here the matrix $A$ is an H-matrix with positive diagonal entries. The corresponding matrix $S$ and its associated permutation matrix $P$ are

$$
S=\left(\begin{array}{cccccc}
2 & 0 & 1 & 0 & -1 & 0 \\
0 & 5 & 1 & -3 & 0 & 0 \\
0 & 0 & 4 & -2 & -3 & 0 \\
0 & -1 & 0 & 2 & 0 & 1 \\
-3 & 0 & 0 & 0 & 5 & 1 \\
-2 & -3 & 0 & 0 & 0 & 4
\end{array}\right), \quad P=I_{6 \times 6}
$$

According to the part (a) of Corollary 1 the matrix $S$ is an H-matrix. Therefore, we exploit its LU factorization for solving the system $S X=Y$. In this case, we obtain

$$
\tilde{x}^{*}=\left(\begin{array}{c}
(1+3 r, 6-2 r) \\
(2+r, 5-2 r) \\
(1+r, 3-r)
\end{array}\right) \text {. }
$$


Example 3: Our last example is devoted to the FSLE $A \tilde{x}=\tilde{b}$, where

$$
A=\left(\begin{array}{ccc}
6 & -1 & -1 \\
-1 & 2 & -1 \\
-1 & -1 & 1
\end{array}\right), \quad \tilde{b}=\left(\begin{array}{l}
(-18+16 r, 8-10 r) \\
(-8+8 r, 6-6 r) \\
(-3+4 r, 8-7 r)
\end{array}\right)
$$

Here the coefficient matrix $A$ is an M-matrix. Since its diagonal entries are positive the corresponding permutation matrix is $P=I_{6 \times 6}$ and

$$
S=\left(\begin{array}{cccccc}
6 & 0 & 0 & 0 & -1 & -1 \\
0 & 2 & 0 & -1 & 0 & -1 \\
0 & 0 & 1 & -1 & -1 & 0 \\
0 & -1 & -1 & 6 & 0 & 0 \\
-1 & 0 & -1 & 0 & 2 & 0 \\
-1 & -1 & 0 & 0 & 0 & 1
\end{array}\right)
$$

As seen, $S$ is of the form (6). According to the part (b) of Corollary 1 the matrix $S$ is an M-matrix and its LU factorization exists. Invoking the LU factorization of $S$ the exact solution of $A \tilde{x}=\tilde{b}$ is obtained as

$$
\tilde{x}^{*}=\left(\begin{array}{l}
(-1+2 r, 2-r) \\
(1+2 r, 4-r) \\
(3+2 r, 8-3 r)
\end{array}\right)
$$

\section{Conclusion}

In this paper, we have verified the existence and uniqueness of a solution to the fuzzy system of linear equations $A \tilde{x}=\tilde{b}$, where $A$ is a crisp H-matrix and $\tilde{b}$ is a fuzzy vector. Our main result in special cases can be applicable for the class of M-matrices and strictly diagonally dominant matrices. Some numerical examples have been shown to illustrate the theoretical results. Future work may focus on generalization of the presented theoretical results for solving fully fuzzy system of linear equations.

\section{Acknowledgements}

The author would like to thank the anonymous referee for the valuable comments and suggestions.

\section{References}

Allahviranloo T 2004 Numerical methods for fuzzy system of linear equations. Appl. Mathematics and Computation 155: 493-502

Allahviranloo T 2005 Successive overrelaxation iterative method for fuzzy system of linear equations. Appl. Mathematics and Computation 162: 189-196

Allahviranloo T, Mikaeilvand N, Kiani NA and Shabestari RH 2008 Signed decomposition of fully fuzzy linear systems. Application and Appl. Math. 3: 77-88

Axelsson O 1996 Iterative solution methods, Cambridge University Press, Cambridge 
Babbar N, Kumar A and Bansal A 2013a Linear programming approach to find the solution of fully fuzzy linear systems with arbitrary fuzzy coefficients. J. Intelligent \& Fuzzy Systems 25: 747-753

Babbar N, Kumar A and Bansal A 2013b Solving fully fuzzy linear system with arbitrary triangular fuzzy numbers $(m, \alpha, \beta)$. Soft Computing 17: 691-702

Dehghan M and Hashemi B 2006 Iterative solution of fuzzy linear systems. Appl. Mathematics and Computation 175: 645-674

Dehghan M, Hashemi B and Ghatee M 2006 Computational methods for solving fully fuzzy linear systems. Appl. Mathematics and Computation 179: 328-343

Dehghan M, Hashemi B and Ghatee M 2007 Solution of the fully fuzzy linear system using iterative techniques. Chaos Solitons Fractals 34: 316-336

Friedman M, Ming M and Kandel A 1998 Fuzzy linear systems. Fuzzy Sets and Systems 96: 201-209

Gong Z, Guo X and Liu K 2014 Approximate solution of dual fuzzy matrix equations. Information Sci. 266: $112-133$

Hadjidimos A 1978 Accelerated overrelaxation method. Mathematics of Computation 32: 149-157

Hashemi M S, Mirnia M K and Shahmorad S 2008 Solving fuzzy linear systems by using the Schur complement when coefficient matrix is an M-matrix. Iranian J. Fuzzy Systems 5: 15-29

Ma M, Friedman M and Kandel A 2000 Duality in fuzzy systems. Fuzzy Sets and Systems 109: 55-58

Meurant G 1999 Computer solution of large linear systems, North-Holland, Amsterdam

Mosleh M, Otadi M and Khanmirzaie A 2009 Decomposition method for solving fully fuzzy linear systems. Iranian J. Optimization 1: 188-198

Nasseri S H, Sohrabi M and Ardil E 2008 Solving fully fuzzy linear systems by use of a certain decomposition of the coefficient matrix. Int. J. Computational and Mathematical Sci. 2: 140-142

Nasseri S H and Sohrabi M 2010 Gram-Schmidt approach for linear system of equations with fuzzy parameters. The J. Mathematics and Comput. Sci. 1: 80-89

Nasseri S H and Zahmatkesh F 2010 Huang method for solving fully fuzzy linear system of equations. The J. Mathematics and Comput. Sci. 1: 1-5

Salkuyeh D K 2011 On the solution of the fuzzy Sylvester matrix equation. Soft Computing 15: 953-961

Senthilkumar P and Rajendran G 2011 New approach to solve symmetric fully fuzzy linear systems. Sādhanā 36: 933-940

Wang K and Zheng B 2007 Block iterative methods for fuzzy linear systems. J. Appl. Mathematics and Computing 25: 119-136 